\title{
Compte rendu du colloque sur l'enseignement des statistiques 2020
}

\author{
Marc-André Goulet ${ }^{\mathrm{a}} \bowtie(\bullet$ \\ ${ }^{a}$ Université Laval
}

\begin{abstract}
La troisième édition du colloque sur l'enseignement des statistiques s'est déroulée le 27 mars 2020 par visioconférence. Le compte rendu du colloque inclut un résumé 1) des présentations de trois conférenciers; 2) des discussions auxquelles les présentations ont mené; et 3) des projets de l'Open Statistics Teaching Initiative (OSTI) pour les prochaines années. Le premier conférencier fut Alexandre Williot, qui proposa de considérer le profil d'apprenant des étudiantes afin d'adapter l'enseignement des statistiques aux besoins de chacun. Le deuxième conférencier fut Bernard Fournier, qui démontra l'utilisation d'un outil de génération de données aléatoires dans le logiciel R. Cet outil permet aux étudiantes de simuler des situations statistiques personnalisées. Enfin, le troisième conférencier fut Alexandre Gellen-Kamel, qui proposa une manière alternative d'enseigner les statistiques en s'inspirant de la pédagogie comportementale, spécifiquement de l'enseignement programmé. Les projets de l'OSTI pour les prochaines années incluent la validation d'un questionnaire sur les profils d'apprenant des étudiantes, la création d'un générateur de données aléatoires dans le logiciel Excel, l'élaboration de vignettes d'enseignement sur des concepts statistiques qui seraient disponibles pour les enseignantes et la réalisation de courtes capsules vidéo destinées aux étudiantes.
\end{abstract} Acting Editor $\square$ Denis Cousineau (Uni-

Keywords $\backsim$ Open Statistics Teaching Initiative; Statistiques.

marc-andre.goulet.3@ulaval.ca

10.20982/tqmp.16.4.p308

\section{Introduction}

La troisième édition du colloque sur l'enseignement des statistiques devait avoir lieu le 27 mars 2020 à Gatineau, en préalable du 42e congrès annuel de la Société québécoise pour la recherche en psychologie (SQRP). À la suite de la décision du gouvernement québécoise d'interdire tout rassemblement public dans l'objectif de mitiger la propagation de la maladie à coronavirus (GOUVERNEMENT DU QUÉBEC, 2020), le colloque s'est tenu en ligne, par visioconférence. Trois conférenciers ont fait une présentation aux participantes et participants du colloque. Le présent compte rendu résume les points principaux de chacune des présentations ainsi que les éléments de discussion qui en ont découlé. Il inclut aussi une énumération des projets prévus dans les prochaines années par l'Open Statistics Teaching Initiative (OSTI).
Le colloque a pour objectif de discuter des défis liés à l'enseignement des statistiques, des méthodes quantitatives, et de la psychométrie, au niveau postsecondaire. Pour la grande majorité des cursus en sciences sociales, les cours de statistiques sont obligatoires et sont une pierre angulaire de la formation des étudiantes. ${ }^{1}$ Ces cours sont toutefois une source d'anxiété pour plusieurs de ces étudiantes (FIELD, 2010). Le rôle des professeures n'est ainsi pas seulement d'enseigner et de développer les habiletés des étudiantes en statistiques, mais aussi d'utiliser des stratégies d'enseignement visant à réduire l'anxiété causée par la matière. Conséquemment, les professeures doivent adapter, voire modifier leur méthode d'enseignement afin de favoriser un sentiment d'efficacité et de réussite chez les étudiantes.

1. Afin d'alléger le texte, le féminin sera utilisé pour désigner la forme féminine et masculine. Par exemple, « les étudiantes » correspond à l'ensemble des étudiantes et des étudiants, et « les professeures » à l'ensemble des professeures et des professeurs. 


\section{Le profil des apprenants}

La première présentation fut donnée par Alexandre Williot (Université Laval) et porta sur la diversité des profils d'apprenant chez les étudiantes. Comme mentionné supra, les cours de statistiques sont une source d'anxiété pour plusieurs étudiantes. Bien que l'attitude des étudiantes par rapport aux statistiques soit corrélée négativement à l'anxiété des statistiques, ce n'en est pas l'unique cause (McINTEE et collègues., soumis). En effet, l'anxiété peut être aussi amplifiée par l'utilisation de stratégies mal-adaptatives face aux situations anxiogènes causées par le cours. Ces stratégies incluent le sentiment de culpabilité et la rumination à la suite d'une situation d'échec. Par surcroît, les étudiantes qui utilisent des stratégies adaptatives pour gérer leur anxiété, telles que l'acceptation et la régulation émotionnelle, sont moins anxieuses par rapport aux cours de statistiques. Finalement, l'anxiété peut être réduite lorsque les besoins psychologiques des étudiantes sont comblés.

Bien que l'attitude par rapport aux statistiques, l'utilisation de stratégies mal-adaptatives / adaptatives, et la satisfaction des besoins psychologiques soient toutes corrélées avec l'anxiété des statistiques, elles ne l'expliquent pas entièrement. Une autre source explicative pourrait être une dissonance entre le profil d'apprenant de l'étudiante et le style d'enseignement de la professeure. Le profil d'apprenant se définit sur deux axes (GUILlEMETTE, s. d.). Le premier axe contraste l'apprentissage par expérience concrète à l'apprentissage par explications théoriques. L'expérience concrète se traduit par des exemples pratiques pour lesquels la solution est donnée par une procédure bien établie. L'apprentissage par explications théoriques mise plutôt sur la compréhension et la logique des procédures. Le deuxième axe contraste l'intégration de la matière par l'expérimentation active à l'intégration par l'explication réflexive. L'expérimentation active met l'accent sur le pragmatisme et sur l'utilisation pratique de la matière vue dans le cours. L'explication réflexive se concentre sur l'observation et l'assimilation de la matière avant de réaliser les activités pratiques.

En positionnant les étudiantes sur ces deux axes, il est possible de déterminer à quel profil d'apprenant elles appartiennent. Un échantillon pilote $(n=23)$ montre que les profils d'apprenant varient beaucoup au sein d'une même classe (Figure 1). Dépendamment de l'approche utilisée par la professeure, il est possible que l'anxiété statistique soit plus prédominante dans un profil d'apprenant par rapport aux autres. Toutefois, pour répondre à cette question de recherche, quelques défis supplémentaires s’imposent. En autres, il faut s'assurer que l'outil utilisé soit validé sur le plan psychométrique avant de mener une étude à grande échelle.

Connaître son profil d'apprenant peut être un bon exercice de prise de conscience pour les étudiantes, une manière de cibler ses forces et ses faiblesses dans le cadre du cours de statistique. Chaque étudiante pourra ainsi développer des stratégies personnelles adaptées à son style d'apprentissage. Pour les professeures, le fait de comprendre que les étudiantes ont des profils d'apprenant différents permet d'offrir des perspectives différentes sur la façon s'enseigner le cours. L'un des plus grands défis pour les professeures est de décentrer la méthode d'enseignement afin d'éviter qu'elle ne soit présentée qu'en congruence avec son propre profil d'apprenant.

\section{Période de questions et de commentaires}

1. Qu'est-ce que les étudiantes ont retiré de l'exercice sur leur profil d'apprenant? Les étudiantes sont encouragées à faire une démarche réflexive sur leur profil d'apprenant, à identifier leurs stratégies actuelles et à considérer des stratégies alternatives qui cadrent mieux avec leurs forces.

2. Est-ce que le choix d'un logiciel d'analyse statistique pour réaliser les exercices dans le cours a un impact sur l'anxiété, en considérant les différents styles d'apprenants? Le choix du logiciel peut assurément affecter l'anxiété ressentie par les étudiantes dans les cours de statistiques. C'est particulièrement le cas pour les logiciels qui misent sur le code et la syntaxe, tels que $\mathrm{R}$ et MatLab.

3. Comment peut-on interpréter les profils des apprenants si le questionnaire n'a pas été validé sur le plan psychométrique? Il est certain qu'il y a du travail à faire pour valider le questionnaire, surtout au niveau de l'approche théorique. C’est une étape nécessaire pour répondre à la question de recherche principale.

4. Y a-t-il une différence entre les cours de statistique du baccalauréat et les cours de statistique du doctorat sur l'apport de l'exercice réflexif sur son profil d'apprenant? Il est difficile de répondre à cette question sans être anecdotique. Habituellement, les étudiantes qui bénéficient le plus de ce type d'exercices sont celles qui sont les plus assidues. Ce nombre risque d'être plus élevé au doctorat qu'au baccalauréat. Ultimement, ce qui va avoir le plus d'impact, c'est le niveau d'implication de l'étudiante dans l'exercice réflexif.

5. Faut-il aborder l'anxiété statistique avec les étudiantes et si oui, comment? C'est important d'en parler avec les étudiantes afin de déstigmatiser l'anxiété et de permettre à celles-ci de constater qu'il s'agit d'un sentiment généralisé. Afin de réduire l'anxiété ressentie dans le cours, la professeure doit rendre les statistiques plus abordables aux étudiantes et s'assurer 
Figure 1 - Répartition des profils d'apprenant chez 23 étudiantes d'un cours de statistiques aux études supérieures. Chaque quadrant correspond à un profil spécifique selon deux axes, soit l'axe expérience-explication et l'axe expérimentation-explicitation.

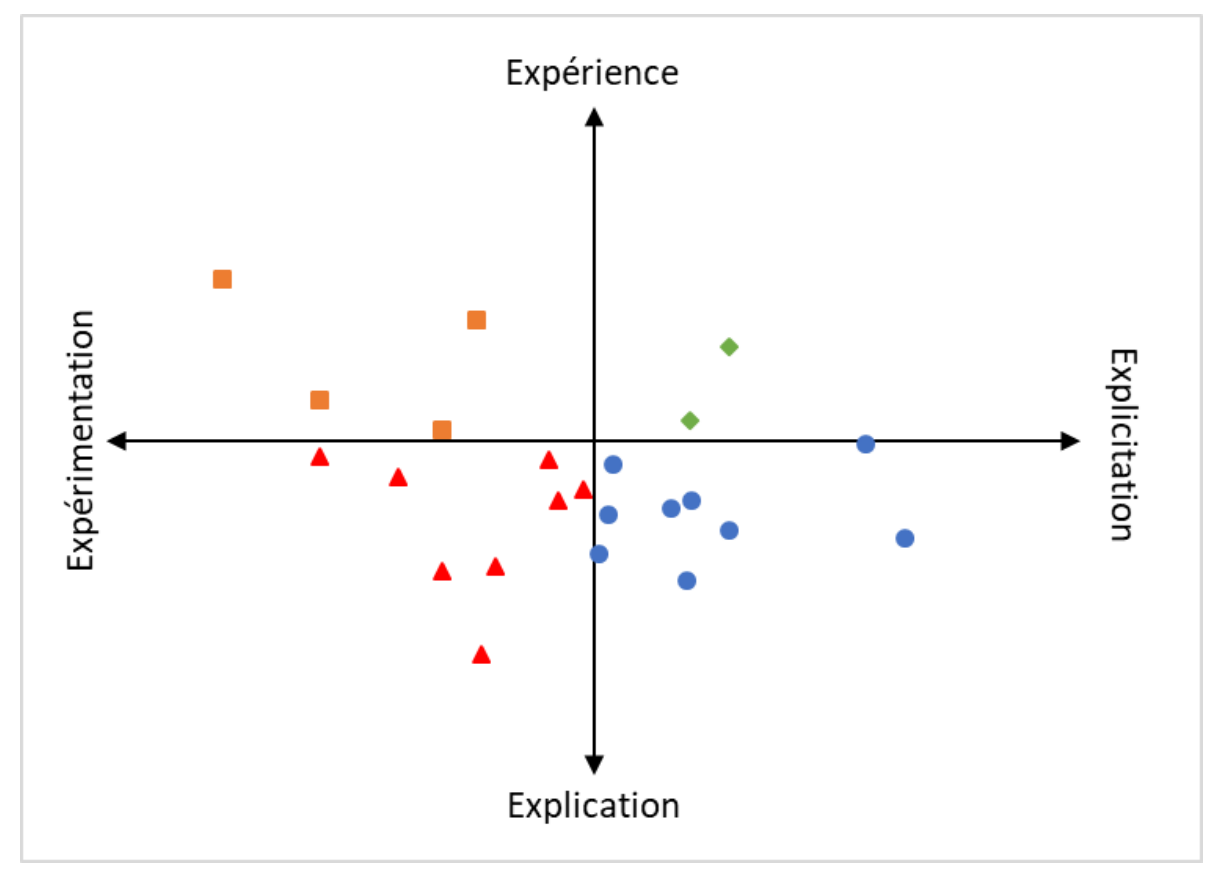

que celles-ci comprennent bien les objectifs du cours. La professeure doit également être honnête avec les étudiantes par rapport à l'anxiété (et parfois partager l'anxiété qu'elle ressent par rapport aux statistiques). Les étudiantes doivent pouvoir mettre un visage humain sur leur professeure.

\section{L’utilisation des données aléatoires}

La deuxième présentation fut donnée par Bernard Fournier (Université de Moncton) et porta sur l'utilisation des données aléatoires. Dans un cours de statistiques, les étudiantes apprennent à prendre des décisions en présence du hasard. En d'autres mots, bien que les procédures d'analyse demeurent les mêmes, les données et les résultats changent constamment. Cette incertitude peut être une source d'anxiété chez les étudiantes. Une manière de réduire cette incertitude est de permettre aux étudiantes de manipuler elles-mêmes les données à partir de générateurs de données aléatoires. En effet, cette technique d'apprentissage mise davantage sur la compréhension des concepts statistiques que sur la mémorisation des procédures, et offre l'opportunité aux étudiantes d'apprendre de façon active dans la salle de classe. Il s'agit, du même coup, de deux recommandations tirées du Guidelines for Assessment and Instruction in Sta- tistics Education (GAISE; Association et collègues., 2005), qui visent à améliorer les méthodes pédagogiques utilisées dans les cours de statistiques.

C'est dans cette optique que l'outil Generator of Random Data (GRD; CALDERINI \& HARDING, 2019) fut développé. GRD est un progiciel téléchargeable pour le logiciel d'analyses statistiques $\mathrm{R}$ qui permet aux étudiantes de simuler aléatoirement différents scénarios afin de mettre en pratique les concepts vus en classe. Après avoir acquis une familiarité avec le progiciel, l'étudiante pourra même créer de nouveaux scénarios et visualiser l'impact de ses décisions sur les données à l'aide de graphiques, comme c'est le cas dans la Figure 2. Le progiciel GRD permet aux étudiantes de voir l'effet de l'échantillonnage sur la distribution des données et d'en induire les concepts statistiques sous-jacents. Par exemple, les étudiantes sont invitées à manipuler la taille d'échantillon et la variance et à observer comment ces deux paramètres influencent l'apparence des distributions, les statistiques calculées (la moyenne et l'erreur-type) et l'interprétation des résultats du test statistique.

Bien que l'utilisation de GRD requière certains prérequis, tels qu'une familiarité avec le logiciel $\mathrm{R}$ et la compréhension de certaines notions de base en statistiques, il s'agit d'un outil simple à utiliser qui permet aux 
FIGURE 2 - Capture d'écran illustrant un exercice pratique réalisé avec le progiciel GRD dans le logiciel R. Dans cet exercice, l'étudiante échantillonne 200 données aléatoires et ne variant que le paramètre d'écart-type dans la population. Les graphiques en bas à droite illustrent l'effet de ce paramètre sur la distribution des données (de gauche à droite, un écart-type de 5, 15, 25 et 35).

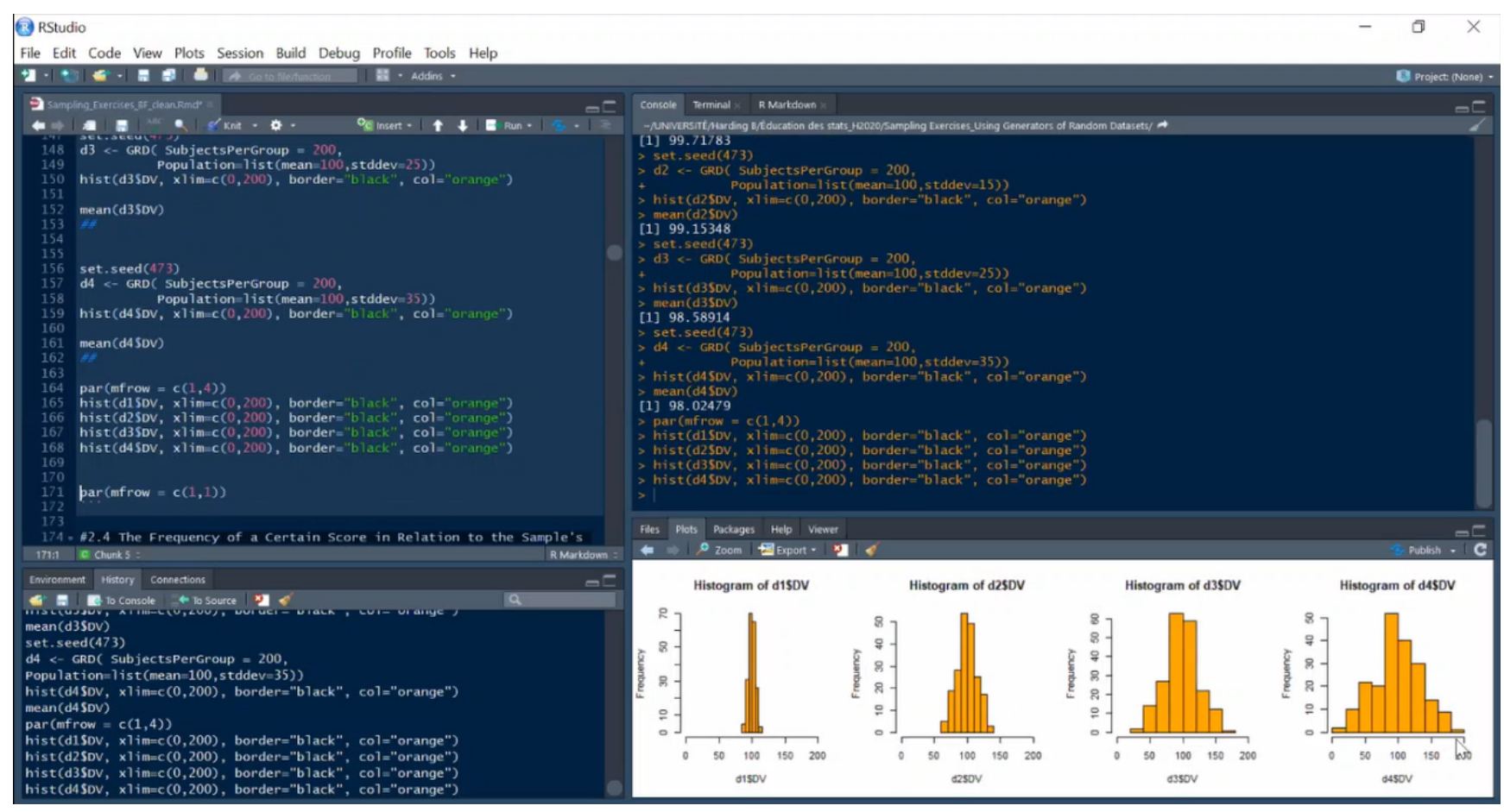

étudiantes de jouer avec les données et de tester par ellesmêmes différents scénarios.

\section{Période de questions et de commentaires}

1. Comment faire pour motiver les étudiantes à développer leur autonomie dans la réalisation de ces exercices, à les motiver à utiliser cet outil ? Le rôle des professeures doit être d'encadrer l'utilisation de l'outil et de l'utiliser en classe lors de présentations magistrales afin de montrer aux étudiantes comment réaliser des simulations à partir de données aléatoires. Aussi, l'outil illustre comment il est possible d'induire les concepts généraux en statistiques à partir de données aléatoires. Une fois que les étudiantes sont familières avec l'outil et avec les résultats générés par celui-ci, elles seront plus enclines à l'utiliser pour répondre à leurs propres questions.

2. Un des défis d'utiliser ce type d'outil est qu'il est difficile d'amener les étudiantes à faire cet apprentissage actif, à prendre le temps de compléter les exercices, souvent par la peur de l'inconnu. Comment régler ce problème? Comme mentionné précédemment, pour engager les étudiantes, il faut que les professeures utilisent l'outil dans un contexte magistral. Une meilleure procédure est de permettre aux étudiantes de réaliser l'exercice en simultané avec leur professeure et de stimuler une discussion autour des différences entre les simulations. Il est aussi important d'assurer une proximité temporelle entre l'apprentissage conceptuel et l'apprentissage actif, entre la théorie et la pratique. Cela permet aux étudiantes de créer plus facilement des liens entre les deux et de mieux intégrer la matière.

\section{L'enseignement programmé}

La troisième et dernière présentation fut donnée par Alexandre Gellen-Kamel (Université du Québec à Montréal) et porta sur un style d'évaluation différent de celui typiquement utilisé dans les cours de statistiques de niveau postsecondaire, soit l'enseignement programmé. Dans son format classique, le cours de statistiques est majoritairement magistral, c'est-à-dire que la matière est présentée sous un angle théorique, laissant peu de temps aux étudiantes pour réaliser des exercices pratiques. De plus, les seules instances d'évaluations pour les étudiantes sont sommatives, sous la forme de deux ou trois exa- 
mens par trimestre. Ces évaluations peuvent représenter une source d'incertitude chez les étudiantes, surtout lorsqu'elles sont incohérentes avec le format d'apprentissage. Ce serait le cas d'une évaluation qui, par exemple, teste les habiletés pratiques, alors que les étudiantes n'ont appris que les concepts théoriques. Enfin, dans le format classique du cours de statistiques, l'objectif ultime n'est pas de s'assurer que les étudiantes maîtrisent parfaitement la matière, mais de classer celles-ci à l'aide des évaluations sommatives.

Cette méthode pédagogique, bien que son utilisation soit répandue, peut s'avérer une source d'anxiété chez les étudiantes et limiter leur sentiment de compétence. L'enseignement programmé propose une pédagogie axée sur la maîtrise de la matière à l'aide d'exercices pratiques tout au long du trimestre. Ainsi, selon ce format (voir Figure 3), la moitié du cours en classe est dédiée à l'application pratique des concepts théoriques enseignés dans l'autre moitié du cours, en recevant une constante rétroaction. Les étudiantes peuvent aussi décider de répéter ces exercices formatifs autant de fois qu'elles le désirent, jusqu'à ce qu'elles les maîtrisent. Une partie de ces exercices sont ensuite utilisés comme outil d'évaluation sommatif, de façon régulière tout au long du trimestre (par exemple, une fois par semaine). Cela assure une cohérence entre ce que les étudiantes ont pratiqué et ce sur quoi elles sont évaluées, ainsi qu'une proximité temporelle entre l'intégration de la matière et l'évaluation de la maîtrise de celle-ci. Les étudiantes ne reçoivent pas de rétroaction durant l'évaluation, sauf à la fin de celui-ci (pourcentage de bonnes réponses), mais peuvent répéter l'évaluation autant de fois qu'elles le souhaitent.

Ce format d'enseignement peut sembler très exigeant, mais permet aux étudiantes de viser une maîtrise parfaite de la matière. Il fut utilisé dans le cadre d'un cours de statistiques au baccalauréat dans lequel on dénombrait 57 étudiantes. Le niveau d'implication des étudiantes varie grandement, certaines profitant des reprises illimitées des évaluations pour maximiser leur maîtrise des exercices. Pour les professeures, ce format de cours peut représenter une très grande charge de travail, surtout à ce qui à trait à la création d'exercices pratiques à fournir aux étudiantes. C'est l'une des raisons pour lesquelles l'enseignement des statistiques ne doit pas être un travail solitaire; au contraire, il doit être fait dans un esprit de collaboration.

\section{Période de questions et de commentaires}

1. Est-ce que ce type de cours peut être monté par une professeure tout en maintenant une charge de travail raisonnable? Initialement, l'enseignement programmé est très exigeant en terme de temps pour les professeures.
Cela dit, une fois que le cours est monté, la majorité du travail est accomplie et n’a pas à être répétée chaque trimestre.

2. L'un des objectifs des évaluations sommatives est d'avoir suffisamment de variabilité autour de la moyenne des notes pour discriminer les étudiantes entre elles, audelà de la formation. Comment peut-on y arriver avec l'enseignement programmé? Il est certain que ce type d'enseignement peut poser certains défis sur le plan administratif, puisque dans ce cours, la note désirée pour chaque étudiante est la maîtrise parfaite de la matière (soit 100\%). Une variante possible est d'utiliser une notation succès-échec plutôt qu'une notation graduée. Par exemple, la note de passage du cours pourrait être de $80 \%$ (maîtrise de $80 \%$ de la matière du cours). Cette éventualité pourrait être envisagée pour certains cours fondamentaux, comme les statistiques, pour lesquels l'objectif premier n'est pas de mettre en rang les étudiantes, mais bien de les former de sorte qu'elles puissent maîtriser les exercices pratiques en statistiques. Une autre possibilité, qui n'est pas incompatible avec la variante succès-échec, est d'impliquer les étudiantes dans le processus d'évaluation.

3. Étant donné la tâche colossale que représente ce type d'enseignement, serait-il envisageable que les professeures de statistiques partagent leurs exercices avec le reste de la communauté des enseignantes en statistiques? La situation idéale est effectivement une grande collaboration entre les professeures. C'est d'ailleurs l'objectif d'un projet en développement, soit celui d'offrir une plateforme web aux professeures pour partager leurs exercices pratiques sous forme de vignette. Il faut toutefois considérer le fait que chaque professeure aborde et présente la matière de façon différente et qu'il n'existe pas d'exercices universels à toutes les approches en statistiques. En effet, certains exercices respectent la procédure suggérée par un manuel de référence, une structure qui n'est pas la même que celle suggérée par un autre manuel de référence. Même avec un tel réseau de collaboration, les professeures devront tout de même s'approprier la matière et modifier les exercices de façon à garder une cohérence avec les autres exercices utilisés dans le cours.

\section{Projets futurs des membres de l'OSTI}

Dans les prochains mois et les prochaines années, les membres de l'OSTI mèneront plusieurs projets sur divers aspects reliés à l'enseignement des statistiques. Les trois présentateurs du présent colloque prévoient enrichir leur domaine de recherche en développant plusieurs outils pour les professeures de statistiques. Ainsi, il y aura un grand effort de validation du questionnaire sur 
Figure 3 - Schéma d'une évaluation utilisant l'enseignement programmé. Les étudiantes peuvent réaliser une série d'exercices formatifs avec de la rétroaction afin de mettre en pratique les notions théoriques vues en classe. Lors de l'évaluation sommative, l'étudiante doit répondre à un échantillon de questions pour lesquelles elle s'est déjà pratiquée, cette fois-ci sans rétroaction. Elle peut répéter cette évaluation autant de fois qu’elle le désire.

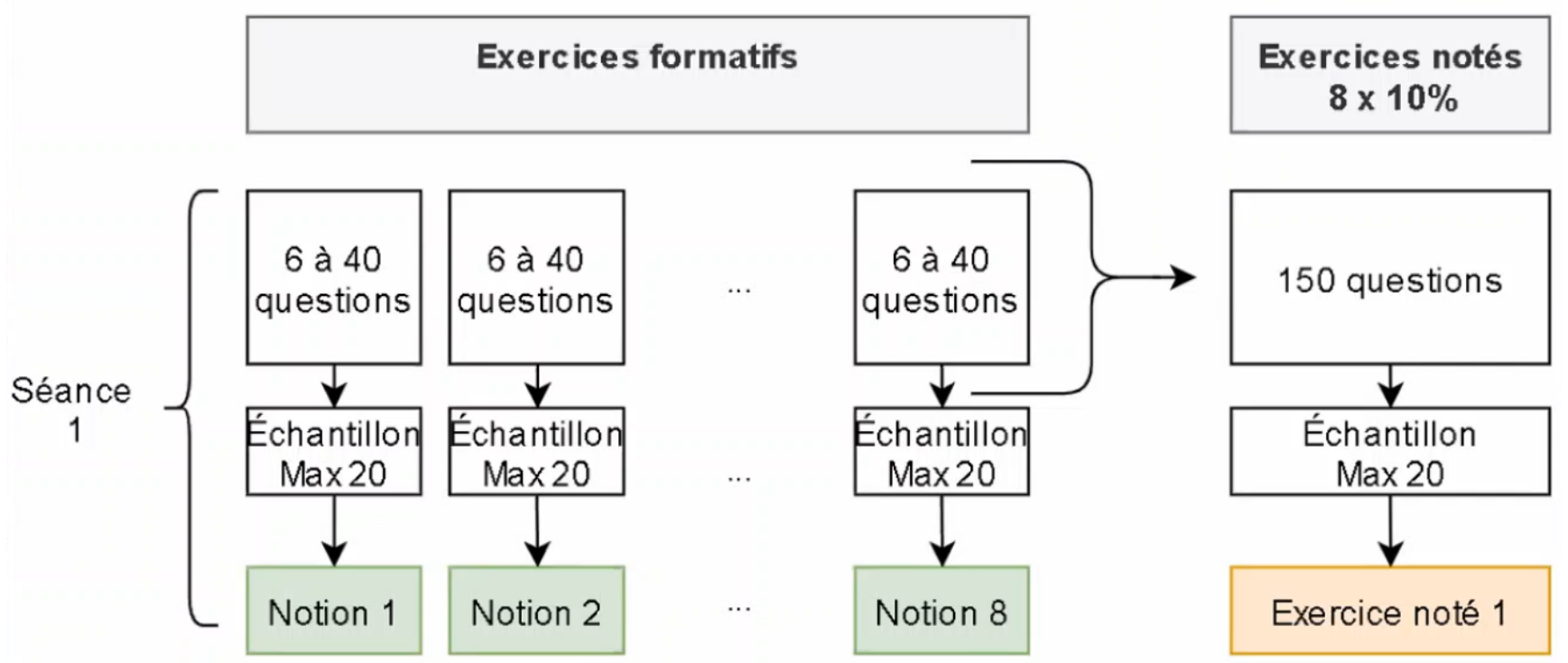

les profils d'apprenant des étudiantes. Ce projet de recherche a comme objectif de doter les chercheuses d'un outil supplémentaire pour déterminer les causes potentielles de l'anxiété statistique. Il y a aussi un projet d'étendre le module GRD à d'autres logiciels d'analyse statistique, particulièrement pour Excel, dont l'utilisation dans les cours de statistiques est répandue. En plus d'être présentement disponible sur R, il existe aussi une version de GRD sur SPSS (HARDing \& Cousineau, 2014, 2015). Enfin, dans le but de favoriser la collaboration entre les professeures, il y a un projet collectif de création de vignettes statistiques, soit un rassemblement d'exercices spécifiques à un sujet en statistiques. Ces vignettes, publiées à la suite d'un processus de revue par les paires, miseraient sur la mise en application de concepts fondamentaux en statistique à l'aide des logiciels d'analyse typiquement utilisés en classe (R, SPSS, Excel).

Les membres du colloque ont également discuté de certaines idées qui pourraient être explorées plus en profondeur dans les prochaines années. Parmi celles-ci, il serait important de s'attarder sur les liens entre les cours théoriques, les exercices pratiques effectués en classe et l'utilisation des statistiques dans un contexte de recherche. Il serait pertinent de s'interroger sur le choix du logiciel d'analyse, particulièrement dans l'optique d'avoir une congruité entre ce que l'étudiante apprend à maîtriser dans son cours de statistique et ce que l'étudiante doit utiliser dans un laboratoire de recherche (à titre de bénévole, d'étudiante à la thèse de spécialisation et/ou en tant qu'étudiante aux études supérieures). Une solution potentielle pourrait être la mise en ligne de courtes capsules vidéo montrant étape par étape comment réaliser une analyse dans un logiciel choisi.

\section{Références}

Association, A. S., Aliaga, M., Cobb, G., Cuff, C., GarFIELD, J., Gould, R., ... WitMER, J. (2005). Guidelines for Assessment and Instruction in Statistics Education [GAISE] College Report. Alexandria, VA : Guidelines for Assessment et Instruction in Statistics Education [GAISE] College Report.

CALDERINI, M. \& HARDING, B. (2019). GRD for R: An intuitive tool for generating random data in R. The Quantitative Methods for Psychology, 15(1), 1-11. doi :10.20982/ tqmp.15.1.p001

FIELD, A. P. (2010). Non-Sadistical Methods for Teaching Statistics. In D. UPTON \& A. TRAPP (Éd.), Teaching Psychology in Higher Education (p. 134-163). Raccoon City, WR : Wiley Online Library. 
GOUVERNEMENT DU QUÉBEC. (2020). Le gouvernement du Québec interdit tout rassemblement intérieur ou extérieur. Récupérée à partir de https : / / www . quebec . ca / premier - ministre / actualites / detail / le - gouvernement - du - quebec - interdit - tout rassemblement-interieur-ou-exterieur/

Guillemette, F. (s. d.). Outil pour mieux se connaître comme apprenant [document non publié]. Université du Québec à Trois-Rivières : Département des sciences de l'éducation.

HARding, B. \& COUSINEAU, D. (2014). GRD : An SPSS extension command for generating random data. The
Quantitative Methods for Psychology, 10(2), 80-94. doi :10.20982/tqmp.10.2.p080

HARDing, B. \& CousineAU, D. (2015). An extended SPSS extension command for generating random data. The Quantitative Methods for Psychology, 11(3), 127-138. doi :10.20982/tqmp.11.3.p127

Mcintee, S. E., Goulet-Pelletier, J. C., Williot, A., DeckLÉGer, E., LAlande, D., CAntinotti, M. \& Cousineau, D. (soumis). (Mal)adaptive cognitions as predictors of statistics anxiety.

\section{Citation}

Goulet, M.-A. (2020). Compte rendu du colloque sur l'enseignement des statistiques 2020. The Quantitative Methods for Psychology, 16(4), 308-314. doi :10.20982/tqmp.16.4.p308

Copyright ( $)$ 2020, GOULET. This is an open-access article distributed under the terms of the Creative Commons Attribution License (CC BY). The use, distribution or reproduction in other forums is permitted, provided the original author(s) or licensor are credited and that the original publication in this journal is cited, in accordance with accepted academic practice. No use, distribution or reproduction is permitted which does not comply with these terms. 